\title{
Certezas y mitos en Educación
}

Dr. Marino Latorre Ariño

\section{RESUMEN}

El artículo trata de identificar y describir algunas certezas -aciertos-- y mitos o errores frecuentes en el imaginario colectivo de los docentes y de la sociedad, en general. Tanto las certezas como los mitos han sido contrastados con los teóricos de la educación y confirmados por mi propia experiencia pedagógica durante décadas de actividad docente, en EBR y en Educación Superior. Describo doce certezas y nueve mitos para insistir en los aspectos positivos de la intervención pedagógica y desvelar los errores en los que se incurre con el deseo de estar al día en innovaciones pedagógicas. La primera y segunda certezas son esenciales: "Sin comprensión no hay aprendizaje"aprendizaje significativo-- y "a aprender se aprende haciendo lo que hay que aprender--aprendizaje funcional--". En cuanto a los mitos en educación señalo, entre otros, "el valor pedagógico de la clase magistral", la creencia que "los exámenes memorísticos miden el aprendizaje", "cuanto más tecnología en el aula, más parendizaje" y "el uso de la paleta de las inteligencias múltiples como una metodología pedagógica".

\section{Introducción}

La educación y la escuela tienen miles de años y los adolescentes y jóvenes que se encuentran estudiando, siempre han sido criticados por la sociedad. El autor chino del Nei Ching, en la colección de textos de la dinastía Chou (1030 al 221 a.C.), recoge las reflexiones del sabio Ch'i Po: "Ahora la gente joven beben y adoptan maneras de vivir muy descuidadas. (...) Solo se preocupan de divertirse; se van a dormir tarde y se levantan a cualquier hora". Un autor de la Edad Media decía: "El joven, a esta edad, poco a nada reflexiona".

Un texto encontrado en una tablilla sumeria de hace 3700 años, narra una disputa entre un padre y un hijo adolescente:

- ¿De dónde vienes?, pregunta el padre.

- De ninguna parte.

- Déjate de tonterías y vete ahora mismo a la escuela y preséntate ante el maestro. 
Espero que lleves los deberes hechos y que no haya ninguna queja de tu comporta-miento. Cuando acabes de la escuela ven directamente a casa sin entretenerte por las calles. ¿Me has entendido?

Platón señalaba que la gente aprendía haciendo; argumentaba contra el sistema existen-te en Grecia. Los críticos romanos se quejaban de que la escuela era irrelevante e inútil. San Agustín abandonó su escuela de retórica en Cartago y se fue a Roma y Milán porque no podía soportar a sus estudiantes, y allí encontró la misma situación. Montaigne se quejaba sobre el sistema francés, Locke del sistema inglés y Einstein sobre el alemán. La escuela, los adolescentes y el aprendizaje, por lo que vemos, han sido un problema a través de la Historia.

En educación, cada año aparecen innovaciones que dicen que van solucionar los problemas que existen en la escuela. Por todas partes surgen demiurgos que poseen la varita mágica para solucionar los problemas de la meducación. Hay buena intención, sin duda, pero a veces hay mucha ingenuidad en los que las proponen y en los que las aceptan, como si se tratara del "bálsamo de Fierabrás"...

Presento algunas de mis creencias en pedagogía, después de muchos años de práctica en el aula, de lecturas de expertos y de mi propia reflexión y práctica pedagógica.

En la segunda parte de este trabajo presento creencias del imaginario colectivo de la educación, que, a mi parecer, son erróneas.

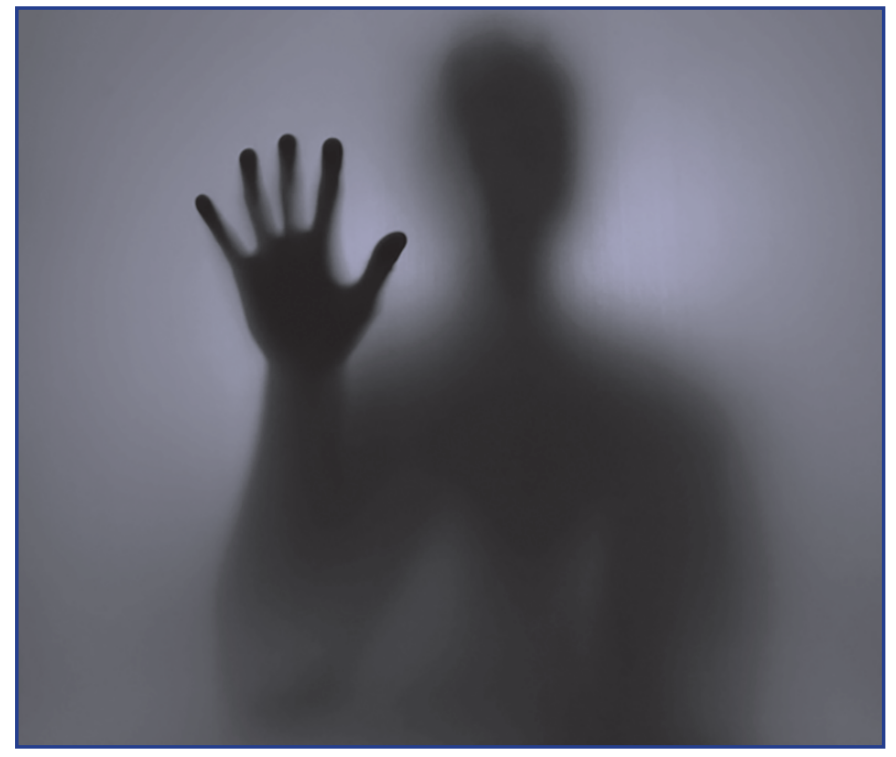

\section{A. Creencias ciertas en educación}

Primera certeza: Si no hay comprensión o razonamiento lógico no hay aprendizaje. Comprender quiere decir asignar significado a algo y para ello hay que identificar, analizar, interpretar, inferir, etc. Se asigna significado a una idea cuando se relaciona, de forma lógica y razonable, con los 
conocimientos previos (aprendizaje significativo de D. Ausubel). Además de utilizar la comprensión para aprender hay que utilizar la emoción, pues "si no hay emoción no hay aprendizaje".

Segunda certeza: Haber aprendido algo es ser capaz de poder utilizar ese aprendizaje en la vida diaria, con sentido y de forma comprensiva, porque "sabemos lo que sabemos hacer y sabemos hacer lo que sabemos" (aprendizaje funcional de J. Dewey). Según Roger C. Schank, el sistema educativo de muchos países --y que ha seguido invariable desde hace siglos hasta nuestros días- se podría resumir así: un profesor entra en clase y habla. Los alumnos, como mucho, toman apuntes. Para comprobar si recuerdan lo que se les dijo en clase el docente les hacen exámenes. Pero poco después de pasar esos exámenes, los estudiantes olvidan todo.

A Schank le gusta preguntar a sus estudiantes: ¿Podrías aprobar ahora, sin estudiar, el exámen de álgebra que pasaste el año pasado? La respuesta es no. ¿Por qué? Entre otras cosas, porque no se puede aprender lo que nos dicen. Los recuerdos siempre van asociados a sorpresas, emociones, retos y acciones. Se aprende haciendo. Y el "aprender haciendo" (J. Dewey) no es un concepto nuevo. Desde la antigüedad los filósofos y científicos han reiterado que es la única forma de aprender. Ya dijo Aristóteles: "Aprender a hacer una cosa se aprende haciéndola".

Tercera certeza: "Aprender haciendo" o "hacer aprendiendo"... "Learning by doing" (Dewey, 2007). ¿Cómo superar la metodología de la clase expositiva o magistral y pasar al formato de aula-taller?

Hoy sabemos, y las ciencias cognitivas lo confirman sin ninguna duda, que la única fuente duradera de aprendizaje es la experiencia, la práctica continua en hacer algo, el learning by doing (aprender haciendo). Pretender mejorar el sistema educativo sin realizar cambios profundos en la metodología y en el aprendizaje, es como querer hacer el tren de alta velocidad manteniendo la tecnología de la máquina de vapor.

Para realizar este cambio hay que responder a estas preguntas: ¿Cómo se organiza el proceso de aprendizaje-enseñanza? ¿Cómo sustituir la clase magistral y posibilitar la inter-disciplinariedad y trans-disciplinariedad entre los cursos? ¿Cómo evaluar a los estudiantes? ¿Qué papel juegan los profesores?

Aprender haciendo implica que cada estudiante tenga un objetivo que conseguir. Los estudiantes deben hacer algo para aprender. En muchos colegios o escuelas y universidades, por desgracia, ¿qué intentan los estudiantes? Sobre todo, aprobar exámenes y conseguir buenas notas. La cuestión es si esa es una habilidad que merezca la pena enseñar y desarrollar.

Un pedagogo decía: "Profesor, cuando vayas a dar una clase no te preguntes iqué tengo que explicar para que los estudiantes lo aporendan?, sino, ¿qué tienen que hacer los estudiantes para aprender lo que yo quiero que aprendan".

Cuarta certeza: Convirtamos la clase en un taller de aprendizaje. El taller entendido como "un lugar donde se trabaja, se produce algo y se transforma algo para ser utilizado" (Ander-Egg, 1999, p. 14). Es un espacio de colaboración y creación de conocimiento, 
$\checkmark$ ya sea el de un mecánico, cambiando, intercambiando piezas de automóviles;

$\checkmark$ el de un ceramista, que con sus manos y un torno crea hermosos diseños a partir de un bloque de arcilla inanimada.

Aprender haciendo es la mejor forma de enseñanza, pero no es fácil trabajar así en clase. Si 25 estudiantes aprenden de esta forma, todos tendrán distintas necesidades y problemas a los que debe enfrentarse el profesor al mismo tiempo. Y todos pueden ir en distintas direcciones y a distintas velocidades, según sus intereses. El problema es lo que se hace en el aula. Cuando hay un aula y un maestro al frente, aprender haciendo es casi imposible. Cambiar esto significa prescindir de las aulas, así como de la idea de un profesor de pie delante de 25 estudiantes, que sabe qué es verdad y la va a contar. Este cambio resulta inconcebible para muchos colegios y universidades.

Quinta certeza: Ser educador es un oficio de artesanos no es un oficio industrial, pues el educador es como:

$\checkmark$ un escultor, que cincela la madera o el mármol para obtener hermosas figuras, volutas y flores;

$\checkmark$ un artesano joyero, engarzando minúsculas piedras preciosas;

$\checkmark$ una modista, cosiendo el vestido soñado de una novia enamorada (Ander-Egg, 1999, p. 12).

Estos artesanos producen objetos hermosos; el educador, por su parte, debe contribuir a tallar y recrear almas únicas, sigulares, diferentes y hermosas.

"Mi deseo con relación al sistema educativo es sencillo: que no aplaste la imaginación que los niños tienen. Eso me parece suficiente. Me gustaría que les dejasen espacio para que sus personalidades encuentren un camino propio y una forma de sobrevivir. De ser así, las escuelas se convertirían en lugares libres y enriquecedores $y$, por consiguiente, la sociedad terminaría por transformarse y evolucionar en la misma dirección" dice el escritor japonés Murakami, (2007).

Sexta certeza: El aprendizaje no se produce cuando alguien -el docente-- quiere enseñar, sino cuando alguien -el estudiante-- quiere y puede aprender. "La verdadera educación se produce de afuera hacia dentro y viceversa. El facilitador -el docente-- debe encontrar el camino de activación". La educación siempre implica la libre aceptación de otros comportamientos, ideas, conocimientos, habilidades, valores, modos de vida, etc. que nos presenta el contexto en el que vivimos; pero eso implica, al mismo tiempo, ser capaz de metabolizar las experiencias personales, los conocimientos ofrecidos y los modelos admirados. A partir de ahí todo es posible.

La evidencia empírica muestra que es imposible enseñar algo a alguien si ese alguien no quiere aprender y no pone esfuerzo de su parte. La motivación es un requisito indispensable para el aprendizaje. Por lo tanto hemos de fijarnos en los mecanismos y motivaciones que guían el aprendizaje de los estudiantes, más que en la enseñanza y en el profesor. La conclusión es que enseñar no es posible si el discente, o mejor el aprendiz, no pone el esfuerzo requerido de su parte y, para ello, ha de estar motivado.

El desarrollo progresivo de las competencias, los valores y las satisfacciones encontradas en el aprendizaje llevan al educando a su propia motivación para la obtención de mejores resultados académicos y para su futura aportación profesional a la sociedad. El romántico Rousseau ya lo 
decía: "Si consigues que el niño quiera aprender, lo hará, sea cual sea el método que utilices". En consecuencia: la principal causa del fracaso escolar es la falta de motivación del estudiante.

Séptima certeza: "El aprendizaje debe contribuir a que las personas sean más felices" (Martín, E.). Una escuela tiene calidad cuando el estudiante aprende en ella, en el momento oportuno y se siente feliz aprendiendo (Bradasky, C., pedagoga argentina). Lo cognitivo y lo afectivo tiene que concretarse en las programaciones para que el docente sepa cómo se van a ir trabajando ambos conceptos a lo largo del curso. "Hoy en día hay demasiados niños brillantes y motivados que se vuelven adultos infelices", porque no han tenido un desarrollo armónico de lo cognitivo y lo emocional.

El éxito de los colegios y la obsesión colectiva por la medición y excelencia académica está, en muchos casos, consumiendo a nuestros niños y profesores, volviéndolos ansiosos, depresivos, frustrados y estresados. Hay colegios británicos que han decido centrándose en el bienestar psicológico de los alumnos como la clave en la educación. Se ha confirmado que al desarrollar técnicas para enfrentar la vida y los desafíos personales, los niños no solo obtienen un buen desarrollo en los ámbitos sociales y personales, sino también en los académicos. El aprendizaje es cuestión de emociones; la experiencia nos dice que se aprende lo que se quiere aprender.

El año 2006, Sir Anthony Seldon, director de un prestigioso colegio público inglés llamado Wellington College, introdujo La felicidad como asignatura de una hora semanal en las clases a partir de los cinco años, donde se les enseña a los niños a vivir. Es impartida por profesores especialistas que tratan temas esenciales que influyen directamente en el bienestar de sus alumnos, bajo la convicción de que el desarrollo de las emociones es igual o más importante que el desarrollo intelectual.

Platón en La República dice que "las leyes harían a una ciudad feliz" y Aristóteles consideraba que la política era la ocupación más noble porque se ocupaba de la felicidad común. Esa fue también la idea de las revoluciones ilustradas. La declaración de Independencia de Estados Unidos (1776) proclama que el fin del gobierno es "alcanzar la seguridad y la felicidad de los ciudadanos". Esto se produce no solo a nivel de escuelas, también de países. En lo profundo de la cordillera del Himalaya, en la frontera entre China e India, se encuentra el Reino de Bután. El primer ministro de Bután, (desde 2013) Tshering Tobgay, comparte la misión que se ha impuesto al gobernar el país y pone la felicidad de sus habitantes y el establecer un estándar mundial para la preservación del medio ambiente, por encima del crecimiento económico. Este enfoque holístico del desarrollo lo llama "Felicidad Interna Bruta", o FIB. En los años 70, el rey de Bután proclamó que la Felicidad Interna Bruta (FIB) es más importante que el Producto Interno Bruto (PIB).

Octava certeza: Nada motiva tanto a los estudiantes y les suscita el deseo de aprender una asignatura, como la pasión y el entusiasmo del docente que la enseña.

Un profesor que con su entusiasmo transmite al estudiante una razonable confianza en que lo hará bien e irá mejorando, suele conseguir que realmente mejore. El efecto Pigmalión afirma que los resultados acompañan a la confianza recibida y a las buenas expectativas ajenas, en mayor medida cuanto más importante sea la persona de referencia para nosotros - padres, profesor, personas a 
quienes admiramos, etc.--. También se aplica la otra cara de la moneda, cuando el profesor aplica una especie de efecto anti-Pigmalión, al rebajar sus expectativas o encasillar a un alumno como inútil, vago o poco inteligente. Es la profecía auto-cumplida.

En neurociencia se habla de "las neuronas espejo" que permiten a las personas percibir los estados de ánimo y sentimientos de otros. No es extraño que el estudiante perciba, por intuición o por efecto de estas neuronas, el estado de ánimo del profesor antes de que pronuncie una sola palabra.

El modelo educativo tradicional fue exitoso durante siglos, pero la sociedad cambió tanto que el modelo ha entrado en crisis. En el pasado "el sistema educativo en la mayor parte del planeta refleja la lógica y las necesidades del mundo industrial, que era racional, organizado y predecible. La organización escolar en casi todos los países se basaba en una currícula con materias específicas y clases de 50 minutos en las que entre 25 y 35 niños de la misma edad estudian siguiendo las órdenes de un profesor. Pero hoy vivimos en un mundo diferente, que se caracteriza por las rápidas transformaciones que afectan a la vida de las personas. El éxito y la felicidad de un joven dependen mucho más que antes de su habilidad para encontrar su pasión por aprender algo e influir en la vida de otros", explicó a Infobae el educador finlandés Pasi Sahlberg, consultor educativo en diferentes instituciones alrededor del planeta Tierra.

Novena certeza: La educación es un arma de contrucción masiva. Paulo Freire decía que "la educación no es capaz de transformar el mundo, pero puede formar personas capaces de transformar el mundo" (Nelson Mandela, Martin Luther King, Ghandi, etc.).

"El objetivo de la educación no se centra únicamente en el éxito de la persona, sino en el interés de formar hombres y mujeres capaces con alto nivel de compromiso social. La educación no debe solo interesarse por el individuo, sino dar las herramientas que le ayuden a compartir con quienes integran la sociedad. La educación debe, además, ayudarlo a descubrir los verdaderos valores de la humanidad (Terre Camacho, 2010).

Décima certeza: La formación de una persona, --como el buen vino o un hermoso diamante--, requiere tiempo, espacio y reposo. El cerebro requiere que se lo use bien, requiere que se piense, que se lea y que se medite. "Algunas cosas sólo necesitan tiempo para que se realicen correctamente... Nueve mamás, no hacen un bebé en un mes" (Buffet, W.).

Juan Amós Comenio (siglo XVII) ya lo dijo: "El arte de la educación es seguir e imitar a la naturaleza". Todo lo que es natural avanza por sí mismo sin detenerse y sin dar saltos, sino de forma gradual. Así hay que proceder en la educación de los niños y jóvenes. La naturaleza se vale del tiempo más favorable, dispone la materia antes de darle forma, realiza sus obras a su tiempo y del mejor modo posible, empieza su obra desde el interior, actúa de forma gradual y sin saltos, cuando empieza una operación la finaliza, elimina lo que es perjudicial, etc. (Comenio, Didáctica Magna).

"En esta época los valores como la brevedad, superficialidad, rapidez y simpleza, son valores absolutos (...) Los valores que desarrolló nuestra sociedad antes fueron la dificultad (para poder sobrellevar y superar los problemas), la lentitud (para reflexionar y no actuar impulsivamente) y la 
profundidad (para saber adentrarse en los problemas). Si se prescinde de estos tres elementos se obtienen reacciones banales, fácilmente manipulables" (Manguel, 2010).

"Como individuos debemos levantar un andamiaje de ideas y pensamientos libres que sirva para oponernos a un sistema de valores nocivo y peligroso basado en conceptos como la rapidez y la eficacia" (Murakami, (2007).

Undécima certeza: El fracaso escolar no es "una catástrofe natural", no es "una fatalidad inherente al desigual reparto de facultades de las personas", sino que es algo racional y social, que está ligado y provocado por el sistema social, económico y cultural en el que nace y vive el estudiante, así como por los intereses de algunas personas de su entorno. El éxito del sistema educativo de un país no se mide por el lugar que ocupan en el ranking de las pruebas PISA sino por el \% de estudiantes que han conseguido los objetivos que el mismo sistema se propuso. En otras palabras, la calidad de un sistema educativo se mide más por el \% de fracaso escolar que tiene, que por el ranking de PISA.

"Las lecciones claves de la experiencia finlandesa --señaló el educador finlandés Pasi Sahlberg-son:

- mantener siempre en un primer plano el trabajo colaborativo y en equipo en lugar de la competencia y de la carrera hacia la cima;

- invertir fuerte y sistemáticamente en equidad educativa más que en incrementar la privatización y la posibilidad de elegir entre distintos colegios; y

- hacer de la docencia una verdadera profesión, insistiendo en que todos los profesores tengan altas credenciales académicas y profesionales, en vez de crear atajos para que puedan dar clase instructores amateurs y poco preparados".

Duodécima certeza: Si queremos certeza que la escuela cambie, el docente tiene que estar en situación de aprendizaje continuo. Los conocimientos son tan fugaces y pasajeros, que lo que fue valioso hace unos pocos años hoy es obsoleto. Un profesional que deja de practicar y estudiar su profesión pronto se convierte en un ex-profesional. "El buen docente es el que continúa aprendiendo, no sólo sobre los temas que enseña sino sobre la propia forma de aprender y enseñar. Lo importante es que el docente reflexione sobre la acción, sobre lo que sucede en el ámbito del aula, lo que sucede más allá de nuestro conocimiento y lo que vemos replicado y reconstruido luego, en cada uno de los trabajos de los estudiantes" (Schön, 1998).

La hipótesis de Seymour Papert, discípulo de Piaget, ilustra bien esta situación: "Si pudiéramos trasladar a un cirujano de 1890 a un quirófano de 2013, entraría en shock y no sabría por dónde empezar. La cantidad de innovación que ha habido en cirugía le impediría siquiera entender lo que sucede. Pero si tomásemos un profesor de instituto o de una universidad de la misma época y lo trasladásemos a una clase de hoy, podría impartirla sin ninguna dificultad". Parece un poco pesimista este señor Papert, ¿tan poco preparados están algunos profesores del siglo XXI? 


\section{B) Mitos o creencias erróneas en educación}

Presento, a continuación, creencias del imaginario colectivo sobre la educación, que, a mi parecer, son erróneas. Se parecen a mitos pedagógicos, que cada cierto tiempo surgen y se aplican sin verificar su efectividad. Los comentarios que hago sobre cada uno de ellos están contrastados con otros autores y hablo desde mi propia reflexión pedagógica; son creencias bien asentadas en la investigación pedagógica, y no improvisadas.

En el comentario de algunos de estos errores seguimos la reflexión de Barajas Casany en una entrevista concedida a Arroyo, C., publicada en el periódico El País, 2013 (España) y realizamos nuestra propia versión y reflexión sobre el tema.

Primera creencia errónea: Enseñar es posible y los profesores enseñan. Galileo dijo: "No podemos enseñar nada a ningún hombre; solo podemos ayudarle a aprenderlo por sí mismo". El docente no enseña, el docente pone al estudiante es situación de que aprenda, pues el aprendizaje tiene lugar en la mente y el corazón del estudiante y no con la metodología del profesor por excelente que sea. En conclusión, podemos afirmar que: El aprendizaje no se produce cuando alguien quiere enseñar (el docente), sino cuando alguien quiere y puede aprender (el estudiante); para ello tiene que estar motivado.

Segunda creencia errónea. La clase magistral como forma prioritaria de organización del aprendizaje. Esta realidad es muy patente en muchas universidades. La clase magistral, a la que sometemos a los estudiantes durante muchos años de su vida, tiene un origen curioso. En los monasterios de la Edad Media, --origen de nuestras escuelas actuales--, un monje que sabia latín, leía un (el) libro de santo Tomás u otros teólogos, la Biblia, los clásicos griegos, etc.; todos estaban escritos por los monjes amanuenses. Al haber pocos monjes y pocos libros se justificaba la lectio (lección o lectura). En esa época, no había mucha discusión posible sobre la interpretación de los textos sagrados. Se aceptaban "los criterios de autoridad". Por lo tanto, "el libro dice la verdad que hay que aprender (memorizar)". Sin más, y hasta aquí hemos llegado.

La clase magistral se puede utilizar cuando se cuenta con un tiempo limitado, cuando las fuentes de información o datos no están al alcance de los estudiantes -que no es el caso en tiempo actuales--, cuando se inicia un tema y se quiere dar una perspectiva general o bien al final del mismo para integrar lo estudiado en un todo coherente. De todos modos tiene que ser en un tiempo breve (unos 30 minutos) a modo de presentación o resumen.

El grado de aprovechamiento de una clase magistral está entre muy poco y nada. La conclusión es que la clase magistral es una metodología educativa ineficiente y caduca. El ego del docente queda muy satisfecho porque ha dado su clase magistral, ha cumplido el temario, piensa que los estudiantes han aprendido, con solo escuchar, todo lo que él sabe y ha explicado, etc., pero la mayor parte de los estudiantes se han aburrido soberanamente, o durante la brillante explicación del docente han estado mandando mensajes, twiteando, wapsapeando, etc. Todo, menos aprender.

En contrapartida ofrecemos el aprendizaje personal y colaborativo, en el que cada estudiante se enfrenta con un contenido (intra-aprendizaje) y después lo comparte con un pequeño grupo (4 
personas) que realizar coordinada y conjuntamente una tarea (inter-aprendizaje). (Roselli, 1999). Para que esta tarea se lleve a cabo de la mejor manera, Ander-Egg (1999) pregona algunos principios pedagógicos que se consideran máximas, como lo son:

$\checkmark$ utilizar siempre una metodología participativa;

$\checkmark$ tanto docente como estudiante deben establecer su relación en torno a una tarea común, donde el docente anime y modere y el estudiante se sienta sujeto de su propio aprendizaje; y, lo que es más importante,

$\checkmark$ en un solo proceso, integrar tres instancias: la docencia, la investigación y la práctica.

Tercera creencia errónea. Las asignaturas son la base para organizar el currículum.

¿Puede alguien concebir un sistema educativo sin asignaturas? Pues bien, no existe ninguna base empírica ni racional para determinar qué es y qué no es una asignatura. Éstas se construyen, normalmente, como compendios secuenciados de temas teóricos que sabe el profesor que imparte la asignatura. La única razón es que "siempre se ha hecho asi". Si la educación tiene como fin preparar para la vida hemos de advertir que la vida profesional real no se organiza por asignaturas, sino que pide resolver problemas mediante la aplicación de complejos procesos de razonamiento y manejo de emociones.

La evidencia empírica nos dice que la transferencia del aprendizaje de una asignatura teórica a la vida real es casi nula. Más aun, relacionar conocimientos dentro de la misma asignatura, en muchos casos, también es casi nula. La conclusión es que organizar el sistema educativo (currículum) mediante asignaturas teóricas es un trabajo inútil de cara al futuro profesional de los estudiantes y, por lo tanto, de la sociedad.

En contrapartida se debe posibilitar la integración disciplinar, la flexibilización curricular, particularmente de los planes de estudio, en aras de formar profesionales más universales y aptos para afrontar las rápidas transformaciones de las competencias y los conocimientos del mundo moderno.

Piaget (1979, pp. 153-171) propone las siguientes dimensiones de integración disciplinar:

$\checkmark$ Multi-disciplinariedad es el nivel inferior de integración, y ocurre cuando alrededor de un problema, caso o situación, se busca información y ayuda en varias disciplinas, sin que dicha interacción contribuya a modificarlas o enriquecerlas. Esta puede ser la primera fase de la constitución de equipos de trabajo interdisciplinario.

$\checkmark$ Inter-disciplinariedad es el segundo nivel de integración disciplinar, en el cual la cooperación entre disciplinas conlleva interacciones reales, es decir, posibilita realizar intercambios y, por consiguiente, un enriquecimiento mutuo. De esta forma llega a lograrse una transformación de los conceptos, las metodologías de investigación y de enseñanza. Implica también que las diferentes disciplinas, al relacionarse, son a la vez, modificadas y pasan a depender unas de otras. 
Trans-disciplinariedad es la etapa superior de integración disciplinar, en donde se llega a la construcción de sistemas teóricos totales, sin fronteras sólidas entre las disciplinas, teniendo objetivos comunes y unificación epistemológica y cultural.

Preguntémonos cuántos científicos y de cuántas disciplinas diferentes trabajaron con el objetivo de llevar el hombre a la Luna, o en el proyecto Manhatan para el programa de desarrollo nuclear en USA, o para la construcción del Concorde, etc.

La conclusión es que organizar el sistema curricular mediante asignaturas teóricas es un trabajo casi inútil de cara al futuro profesional de los estudiantes y, por lo tanto, de la sociedad.

Cuarta creencia errónea. Los exámenes miden el aprendizaje. La evidencia empírica dice que nadie sería capaz de aprobar dos meses después el mismo examen que aprobó en su día, sin haberlo vuelto a estudiar. Y que un profesor de Historia de secundaria o de universidad sería incapaz de aprobar el último examen de Física que estudió cuando era estudiante. Lo mismo le ocurriría al profesor de Física respecto de la Historia. Si los propios profesores son incapaces de aprobar los exámenes de otra asignatura, ¿cómo podemos exigir a los estudiantes que los aprueben? Si el examen es importante y su contenido también, ¿no debería ser algo que cualquier adulto supiera hacer de forma natural?

La evidencia empírica nos dice que la memorización, en la que se basan la mayoría de los exámenes, tiene un efecto muy limitado en nuestra mente. Nuestro cerebro no está preparado para retener grandes cantidades de información. Y realmente no lo necesitamos. Schleicher, A. (2016) dice que "la mayor parte de los exámenes escolares que responden los estudiantes de América Latina se pueden responder rápidamente con ayuda de un teléfono inteligente".

"Me parece que el objetivo de muchos profesores es meter conocimientos en la cabeza de los estudiantes según lo que dicte el manual de turno y enseñarles una técnica para superar los exámenes y las distintas pruebas de acceso a las que han de enfrentarse a lo largo de todo el período educativo" (Murakami, H. 2007).

El mismo autor japonés insiste en el despropósito y absurdidad del abuso de la memorización mecánica: "No me parecía en absoluto útil memorizar fechas de acontecimientos del pasado, archivar palabras del inglés como si yo fuera una máquina. Los conocimientos aprendidos mecánicamente, y no como un todo sistémico, acaban por desaparecer y se quedan por ahí enterrados en alguna parte, en un lugar que podríamos considerar la tumba del conocimiento".

La conclusión es que la memorización excesiva es un esfuerzo inútil, y los exámenes tradicionales (memorísticos) también. Hay muchos estudiantes que estudian para aprobar e inmediatamente olvidar lo aprendido y otros pero son los menos, que estudian para aprender a vivir, utilizar lo aprendido y ser buenos profesionales.

Lo que pretenden medir los exámenes son los aprendizajes de los estudiantes; pero hoy en día,-en que los conocimientos son infinitos y perecederos--, el verdadero aprendizaje consiste en desarrollar en el estudiante habilidades (capacidades-destrezas) y emociones -valores y actitudes-- 
como herramientas mentales y emocionales que le permitan aprender cualquier contenido durante toda la vida; este es el concepto de aprender a aprender (Beltrán 1993).

En consecuencia ipor qué no proponer exámenes en los que los estudiantes demuestren qué saben hacer con los conocimientos que poseen? Si de esto se trata, ipor qué no van a poder utilizar durante el examen todos los materiales que necesiten para demostrar lo que saben y lo que saben hacer con lo que saben?

"No comprendo por qué la escuela excomulga a los copiadores-plagiadores, --a quienes copian durante los exámenes--, si la escuela toda es un templo a la copia; a la copia cultural. ¿Quién copia más que un profesor?" (de Zubiría, M. 1994, p. 140).

Quinta creencia errónea: Las calificaciones finales de una asignatura se obtienen haciendo promedios de las notas parciales obtenidas.

La pedagogía del siglo XXI pretende desarrollar en los estudiantes competencias personalessistémicas, interpersonales e instrumentales. En la Sociedad del conocimiento entendemos por competencia una adecuada integración, de forma sinérgica, de los elementos siguientes: capacidades-destrezas (habilidades o herramientas mentales cognitivas), valores-actitudes (tonalidades afectivas de la persona), dominio de contenidos sistémicos y sintéticos (formas de saber, episteme) y manejo de métodos de aprendizaje (formas de saber hacer, epitedeume); todo ello aplicado, de forma práctica y activa, para resolver problemas de la vida y en el trabajo profesional, en contextos determinados. La competencia surge de la intersección de tres elementos: conocimientos, habilidades y actitudes; cuanto mayor sea el área de intersección mayor será la competencia.

Como vemos las competencias nos sitúan en el marco de lo que un estudiante debe saber, saber hacer, saber ser y saber convivir.

Las competencias son capacidades en acción que permiten responder a las exigencias individuales o sociales para realizar una actividad según los estándares de desempeño asignados; los estándares están expresados y concretados en los niveles de desempeño y definen el nivel que se espera que alcancen todos los estudiantes al finalizar los distintos ciclos de un programa, curso o carrera.

¿Cómo se logra evidenciar lo aprendido? A través de los desempeños de los estudiantes, que son descripciones específicas de lo que saben hacer y hacen los estudiantes en relación a los niveles de desarrollo de las competencias (niveles de desempeño) en el nivel que les corresponda.

El desempeño del estudiante se expresa a través de la evidencia de lo que el estudiante es capaz de hacer (habilidades cognitivas o manuales) con los contenidos que trabaja en una asignatura y grado y la actitud con la que lo hace (competencia). Los indicadores permiten comprobar, a través de evidencias, si el trabajo realizado por el estudiante concuerda con el resultado esperado, es decir, cómo utiliza la destreza y la actitud cuando maneja los contenidos, el estudiante.

En consecuencia la evaluación tendrá que comprobar en qué grado ha desarrollado las competencias según el nivel de desempeño esperado al finalizar el proceso; no importa los errores del proceso 
de aprendizaje -el error es parte del aprendizaje--. Al concertista se le valora por la interpretación ante el público el día del concierto, no por los errores que cometió mientras estudiaba la partitura.

En consecuencia, cuando se evalúa por competencias, la calificación que debe tener el estudiante al finalizar el proceso es la del trabajo final. Cuando la finalidad de la educación era aprender contenidos, el estudiante podía saber unos e ignorar otros, y era necesario hacer promedios para poder calificar.

En competencias no es así, pues al finalizar el proceso de aprendizaje el estudiante ha desarrollado hasta un cierto grado las competencias; conociendo este grado de desarrollo se califica y acabó el proceso.

Las pruebas de proceso realizadas durante el tiempo que dura el aprendizaje solo sirven para dar orientaciones al estudiante y al docente sobre la marcha del proceso de aprendizaje-enseñanza y tomar las medidas correctivas adecuadas.

Sexta creencia errónea. El sistema educativo enseña a entender el mundo. La conclusión es que lo que sucede a veces en el aula, --cuando los estudiantes realizan una actividad ritualizada y descontextualizada del mundo real--, no tiene nada que ver con la vida real y, por lo tanto, es muy difícil que sirva para "entender el mundo y la vida". En el aula sucede algo parecido a lo que vemos en las películas de cine; lo que sucede en las películas es una cosa y la vida real es otra muy diferente.

La realidad es que los procesos pedagógicos que se realizan hoy en muchas aulas (clase magistral del profesor, exposición y estudio de teorías des-contextualizadas y exámenes por asignaturas, etc.) están completamente obsoletos y no han variado en los últimos 500 años.

El escritor japonés Haruki Murakami (2007), hablando de su propia experiencia, dice que "lo más importante que aprendí en la escuela es que las cosas más importantes no se pueden aprender allí"... "Cuando mi vida de estudiante llegó a su fin, estaba tan inmensamente aburrido que lo único que quería era no aburrirme nunca más en toda mi vida. Me lo propuse con todas mis fuerzas, pero en esta vida el aburrimiento parece que cae del cielo y brota de la nada". Para Murakami la lectura fue su gran escuela; en ese lugar aprendió las cosas importantes de la vida, a su aire, sin competir con nadie para "alcanzar el primer puesto de ningún ranking".

Séptima creencia errónea: Cuanto más tecnología digital se utiliza en el aula se producen más y mejores aprendizajes. Partimos del presupuesto que la tecnología digital ha llegado al planeta Tierra para quedarse; pero también hemos de afirmar que la tecnología, por sí sola, no resuelve los problemas de la educación. No hay que tecnolgizar la pedagogía sino que hay que pedagogizar la tecnología. Además no es una tesis comprobada que la mucha tecnología en el aula produzca mejores aprendizajes. La tecnología amplía los buenos resultados y amplifica los malos resultados... Todo depende de la utilización adecuada de la misma; eso es obra del docente. Por ser la tecnología un arma poderosa y peligrosa, hay que saberla "utilizar adecuadamente" (Schleicher, A. 2016). 
En el ámbito de la educación lo primero que hay que preguntarse es el para qué -la pregunta educativa-- y después viene el cómo, --la pregunta metodológica-- es decir, qué tecnología se puede y se debe utilizar en cada momento de la intervención educativa, pues la tecnología es una herramienta, no un fin.

Lo esencial es que la educación prepare personas capaces de "producir conocimiento a partir de la información de que se dispone", (McCarthy, M. J., 1991). Es cierto que la tecnología proporciona más posibilidades para recibir información, pero la información recibida será útil y podrá compartirse, añadiéndole un valor agregado, si el sujeto (el nodo que recibe la información) es capaz de producir conocimiento con la información que recibe; esto se producirá siempre y cuando el que recibe la información posea herramien-tas mentales y actitudinales adecuadas para poderla utilizar.

Este es el gran desafío de la escuela y de la universidad. "Podemos tener tecnología cien y pensamiento cero"... Un colegio puede ser un bosque de tecnología y simultáneamente un desierto en humanidad. Para muchos estudiantes el "navegar por Internet" se convierte en "naufragar en Internet...". Y peor aun no el aspecto social: "Cuanto más conectado, más desconectado".

El educador es irreemplazable, pues una máquina puede aportar datos, información, etc., pero nunca el calor humano, el afecto, la acogida, la comprensión, la motivación y la mediación adecuada, cuando sea precisa. Una educación sin el encuentro personal estudiante-docente, se puede convertir en un panteón de verdades. En consecuencia si "un profesor puede ser sustituido por una máquina, debe ser urgentemente sustituido" (Arthur Clark).

Las alumnas del instituto femenino Stroud de Gloucestershire (Inglaterra) han pedido la desconexión digital en el aula; a partir de septiembre (2017), no podrán utilizar ninguno de estos dispositivos en las instalaciones del centro, salvo algunas excepciones. En un primer momento les chocó la decisión tomada, pero poco a poco las chicas han entendido y han aplaudido la decisión.

A principios de 2017, los responsables del centro realizaron una encuesta sobre este asunto y descubrieron que el $75 \%$ del alumnado revisaba continuamente sus redes sociales y que el $50 \%$ incluso se dormía con el teléfono en la mano. Pero lo más sorprendente es que más de la mitad de las chicas de entre 11 y 14 años aseguraron que les gustaría "tener más control sobre el uso de las redes sociales".

"Las chicas quieren más control porque no saben cómo conseguirlo. Y necesitamos ayudarlas. El colegio debe ser un lugar en el que se pueda aprender, divertirse y tener amigos auténticos, no es un lugar para hablar a través de Snapchat", asegura Cindi Pride, la directora del centro, en declaraciones concedidas a The Guardian.

Octava creencia errónea: Utilizar como método de enseñanza "la paleta de las inteligencias múltiples" (IM) produce mejores aprendizajes.

Hay muchos autores que han afirmado que no existe evidencia de que la aplicación el "método de las IM" en el aula produzca mejores aprendizajes. (Hodge, 2005; Waterhouse, 2006). Se han 
hecho muchos estudios y no se ha logrado demostrar la relación causa efecto entre el uso de "la paleta de la IM" y la mejora de los aprendizajes (Hodge, 2005). El propio Gardner reconoce, como vemos más adelante, la falta de evidencia existente y llega a expresar su descontento con la forma como se ha empleado su teoría en la práctica pedagógica (prólogo del libro de Kornhaber et al., 2004).

H. Gardner (2001) dice en su libro "La inteligencia reformulada" (2001) que, "desde que publicó, en los años 90, el libro Frames of Mind --Inteligencias Múltiples (IM)-- se ha encontrado con centenares de reinterpretaciones diferentes de la teoría de las posibles aplicaciones en el campo de la educación. A la vista de lo que he leído y de mis propias observaciones, he identificado varios mitos sobre las IM". En ese libro desmonta varios de ellos.

\section{Presentamos algunos mitos:}

10 mito: Que existan test contrastados y validados para determinar las inteligencias múltiples. Diseñar una batería d etest para medir las inteligencias múltiples no es coherente con la teoría propuesta por Gardner.

20 mito: Una inteligencia es lo mismo que una disciplina o asignatura. Una inteligencia se basa en potencialidades y capacidades de carácter biológico y psicológico. No se debe confundir con ninguna área o disciplina, que son esferas de actividad humana construidas socialmente.

La confusión surge, --según el propio Gardner--, por la falta de claridad en el concepto de inteligencia explicado en su primer trabajo. Ahora afirma que una inteligencia es una potencialidad biopsicológica que tiene la especie humana. Esta potencialidad se puede desarrollar, en mayor o menor medida, como consecuencia de las condiciones biogenéticas, las experiencias personales, los factores culturales y las motivaciones de cada persona.

$3^{\circ}$ mito: Una inteligencia es lo mismo que un estilo de aprendizaje, un estilo cognitivo o un estilo de trabajo. Una inteligencia es una capacidad para aprender determinados contenidos específicos. El estilo designa un enfoque o manera de hacer general que una persona puede aplicar por igual para aprender contenidos vaiados e indefinidos. El estilo de aprendizaje es personal; en consecuencia, se puede hablar de estilos de parendizaje pero no de estilos de inteligencia.

40 mito: Existe un método educativo que se base en la teoría IM. La teoría de las IM no supone ninguna fórmula o método educativo. Los educadores son quienes se encuentran en la mejor posición para determinar hasta qué punto deben guiarse por la teoría de las IM en su práctica cotidiana en el aula.

La postura de Gardner en la intervención pedagógica en el aula se refleja en este proverbio chino: "Dejad que florezcan cien flores". Así pues, para favorecer el desarrollo de las diversas inteligencias debemos ofrecer espacios con recursos y estímulos variados donde cada uno pueda descubrir y potenciar su manera personal de aprender.

En este sentido, Howard Gardner y su equipo de investigación, han realizado el Proyecto Spectrum, en el que se ofrecen diferentes recursos a los alumnos de Infantil y Primaria para 
ver en qué inteligencia tienen más facilidad y de qué manera aprenden, y así poder adaptar el currículum.

Gardner identifica en su libro "La inteligencia reformulada" (2001) algunas aplicaciones que considera que no son correctas:

1. Intentar enseñar todos los conceptos de todas las materias empleando todas las inteligencias. Es indudable que la mayoría de los temas se pueden abordar de diversas maneras, pero aplicar un enfoque tan amplio y disperso para cada tema constituye una pérdida de tiempo y de esfuerzo.

2. Creer que ciertos movimientos activan o ejercitan unas inteligencias concretas. He visto clases donde se anima a los niños a mover los brazos o a corretear, bajo el supuesto de que estos ejercicios refuerzan la inteligencia corporal-kinestésica. No quiero decir que el ejercicio sea malo, sino sólo que los movimientos musculares al azar, no tienen nada que ver con el cultivo de la mente (y puede que del cuerpo).

3. Etiquetar a las personas en función de "sus inteligencias". Emplear la terminología de las distintas inteligencias para identificar a las personas es un agradable juego de salón. De manera informal, se puede decir que es "muy lingüístico" o "matemático", etc. Ese no es un lenguaje ni educativo ni científico; es un lenguaje coloquial y a veces irrespetuoso. Cuando se etiqueta a alguien de esta manera, se acaba pensando que sólo es capaz de trabajar o de aprender de ciertas maneras, cosa que casi nunca es verdad.

Gardner concluye diciendo: "Por todas estas razones, me resisto a dar una orientación para las llamadas Escuelas IM. En cambio, considero que la teoría de las IM respalda totalmente tres proposiciones fundamentales:

$\checkmark$ que no somos todos iguales,

$\checkmark$ que no tenemos todos la misma inteligencia, y cada estudiante tiene habilidades determinadas, que el docente debe descubrir y potenciar.

$\checkmark$ que la educación actúa con más eficacia si se tienen en cuenta estas diferencias en lugar de negarlas o ignorarlas.

Tomar en serio estas diferencias se encuentra en el corazón de la perspectiva de las IM. En el plano teórico, esto significa que no sirve de gran cosa colocar a todas las personas en una sola dimensión intelectual. En el plano práctico, significa que los enfoques educativos uniformes sólo son óptimos para un porcentaje pequeño de niños. Es conveniente emplear materiales, recursos pedagógicos y actividades que sean ricos y variados y respondan a la diversidad del alumnado dentro del aula.

Nuestro aprendizaje depende más de las herramientas mentales y emocionales de que disponemos, del esfuerzo que se dedica, de las experiencias previas en la vida, etc. que de lo bueno o malo que sea el profesor, de las condiciones materiales del colegio, de los recursos pedagógicos utilizados y del talento natural. Como hemos dicho más arriba: "Hoy en día hay 
demasiados niños brillantes y motivados que se vuelven adultos infelices por no tener un desarrollo integral de su persona".

Por este motivo no hay una marca o título con que podamos designar a escuelas de IM, sino que las escuelas que asumen la teoría, definen una metodología según sus objetivos educativos.

Novena creencia errónea: La estimulación temprana abundante en los primeros años de vida produce niños más inteligentes. La doctora María L. Ageitos, cónsultora de Unicef, reafirma que todo lo que recibe un niño en sus primeros años de vida es clave para su futuro desarrollo y su desempeño como individuo, pero que "lo que ayuda al niño a crecer y a desarrollarse está íntimamente relacionado con los ámbitos de crianza. Los tiempos cambian, pero aún hoy lo más importante sigue siendo la relación afectiva, el intercambio, el compartir, el sentido de pertenencia".

Hay padres que quieren que sus hijos sobresalgan del resto de niños para poder afirmar que son muy inteligentes, superdotados, genios, etc. y para ello los sobre estimulan. Pretenden que sepan leer a los dos o tres años, que toquen el piano a los cuatro años (a ejemplo de Mozart). "Vivimos en una sociedad de consumo, donde es importante competir y sobresalir; por eso, la estimulación se ha convertido en una herramienta para fabricar genios", dice María L. Ageitos. Pero no hay nada más lejos de la realidad. iSi al niño no le gusta lo que hace, la estimulación, es una tortura!

Estos padres no saben que para aprender se necesita el desarrollo físico del cerebro; que haya conexiones neuronales adecuadas y eso tendrá lugar cuando el niño establezca vínculos afectivos con la mamá y su entorno, cuando se desarrolle su sicomotricidad, sus sentidos, etc. de forma adecuada. Ese es el mejor estímulo para que un niño crezca sano y desarrolle su inteligencia. "Hay tres cosas que recomendamos los pediatras, que son: tomar al niño en brazos, el mimo y la teta; tres cosas básicas y esenciales para los primeros meses", dice María Ageitos.

No se trata, pues, de proporcionar al niño una gran cantidad de sensaciones y de objetos destinados a su entretenimiento y que sirvan como estímulo. El mejor estímulo es que el niño se sienta querido, acompañado por sus padres para ver lugares, imágenes variadas, panoramas, oír sonidos diversos, etc.; estimularlos es ayudarlos a descubrir el mundo, a probar y hacer cosas nuevas. Es demostrarles que utilizando sus cinco sentidos pueden pasar ratos muy entretenidos. La forma natural de aprender el niño es a través del juego, y para jugar no necesita los juguetes más caros y modernos. El niño suele divertirse más con los objetos que encuentra diariamente.

No olvidemos que el niño no puede crecer solito, como una flor; necesita compañía, amor, cercanía cariñosa de sus padres y seres queridos. Requiere tiempos largos de estar con los que quiere y le quieren; oigo a padres que hablan de "tiempos intensos pero cortos de relación" con sus hijos; no se trata de tiempos intensos sino tiempos prolongados, aunque ello implique tiempos de aburrimiento.

Presento el documento preparado el año 2004 por la Oficina Regional de Unicef para las Américas. 


\section{Decálogo básico del desarrollo psicosocial infantil}

1. Para el niño es fundamental el contacto estrecho con los padres antes y en el momento de nacer.

2. El niño necesita establecer un vínculo o relación de afecto y amor con sus padres o las personas que lo cuidan.

3. El niño necesita un intercambio con su medio a través del lenguaje y del juego.

4. El niño necesita hacer las cosas por sí mismo para alcanzar un grado adecuado de autonomía o independencia.

5. El niño necesita la valoración positiva para tener una buena autoestima y confianza en sí mismo.

6. El niño necesita tener un mínimo de seguridad y estabilidad.

7. El niño necesita poder expresar sus emociones y sentimientos sin temor a ser reprimido 0 castigado.

8. Cada niño es distinto, tiene su propio temperamento y su propio ritmo; no todos los niños aprenden con la misma rapidez.

9. Las familias que apoyan a los niños, los estimulan y son cariñosas con ellos y tienen niños más sanos y felices.

10. Los padres y adultos deben evitar golpear, maltratar, asustar, descalifi-car o engañar a los niños. Un ambiente de irritación, violencia o inestabilidad prolongada es perjudicial para el desarrollo infantil. 


\section{REFERENCIAS}

Ander-Egg, E. (1999). El taller: una alternativa de renovación pedagógica. Buenos Aires: Magisterio del Río de la Plata.

Arroyo, C. (2013). Reinventemos nuestra educación porque se basa en creencias erróneas. Entrevista a Barajas, S. Blog Sociedad. El País. Recuperado de: http://blogs.elpais.com/ ayuda-al-estudiante/2013/11/reinventemos-nuestra-educacion-porque-se-basa-encreencias-erroneas.html

Beltrán Llera, J. (1993). Procesos, estrategtias y técnicas de aprendizaje. Madrid: Síntesis.

Dewey, J. (2007). Cómo pensamos. Barcelona: Paidós.

Gardner, H. (2001). La inteligencia reformulada. Barcelona: Paidós

Hodge, E. (2005). A best-evidence synthesis of the relationship of multiple intelli-gence instructional approaches and student achievement indicators in secondary school classrooms (Tesis doctoral). Recuperado de: http://digitalcommons.cedarville.edu/cgi/viewcontent. cgi?article $=1009 \&$ context $=$ education_theses

Kornhaber, M. L., Fierros, E. G., \& Veenema, S. (2004). Multiple intelligences: Best ideas from research and practice. Needham Heights, MA: Allyn \& Bacon Publishers.

Latorre, M. (2016). Diseño curricular por capacidades y competencias en educación superior. Lima: Universidad Champagnat.

Manguel, A. (2010). La ciudad de las palabras. Buenos Aires: RBA.

Martín, E. (2009). "2009-2025: alumnos competentes", en "Escuelas con visión" Revista Educación y Gestión y FERE-CECA. Año V - No 29.

Murakami (2007). De qué hablo cuando hablo de escribir. Barcelona: Tusquets.

Piaget, J. La epistemología de las relaciones interdisciplinarias, en: L. Apostel, G. Berger, A. Briggs y G. Michaud (1979). Interdisciplinariedad. Problemas de la enseñanza y de la investigación en la Universidades. México: Asociación Nacional de Universidades e Institutos de Enseñanza Superior.

Roselli, N. (1999). La construcción sociocognitiva entre iguales. Rosario, Argentina: IRICE.

Schön, D. (1998). La formación de profesionales reflexivos. Madrid: Paidós.

Schleicher, A. (2016). Conferencia. Lima, 20/04/2016.

Terre Camacho, 0. (2010). Recuperado de: www.orlandoterre.com 
UNICEF (2004). Decálogo básico del desarrollo psicosocial infantil. Recuperado de: http:// puertocrianza.com/blog/formacion-del-nino-2/decalogo-del-desarrollo-psicosocial-infantil/

Zubiría, M. (1994). Tratado de pedagogía conceptual. Pensamiento y aprendizaje. Santa Fe de Bogotá, Colombia: Fundación Merani.

Waterhouse, L. (2006). Multiple intelligence, the Mozart effect, and the emotional intelligence: A critical review. Educational Psychologist, 41, 207-225. 